\title{
SIMULATION OF ACTIVATION AND PROPAGATION DELAY DURING TRIPOLAR NEURAL STIMULATION
}

\author{
Eleanor V. Goodall, Member, IEEE, L. Martin Kosterman, \\ Johannes J. Struijk, Member, IEEE, and Jan Holsheimer, \\ Biomedical Engineering Division, Dept. of Electrical Engineering, \\ University of Twente, P.O.Box 217, 7500 AE Enschede, the Netherlands
}

\begin{abstract}
Computer simulations were performed to investigate the influence of stimulus amplitude on cathodal activation delay, propagation delay and blocking during stimulation with a tripolar cuff electrode. Activation and propagation delays were combined in a total delay term which was minimized between the excitation and blocking thresholds.
\end{abstract}

\section{INTRODUCTION}

Cuff electrodes with tripolar contact arrangements can be used to selectively activate small nerve fibers by combining cathodal excitation with anodal block of larger fibers [1],[2]. Action potential (AP) propagation may be delayed near the anode at stimulus levels too low to produce blocking [3]. The temporal relationship of activity from fibers of different sizes produced by tripolar stimulation can be rather complex. Simulations were performed to obtain a better understanding of the influence of stimulus amplitude and fiber size on activation and propagation delay during stimulation with a tripolar cuff electrode. We expect that this will aid in interpreting compound neural signals recorded from stimulated nerves, which can be used as a means of assessing the selectivity of electrical stimulation.

\section{METHODS}

A two part computer model was used in our simulations [4]. The potential field within a 3-dimensional volume conductor model of the fascicle, surrounding tissues, and cuff electrode was computed by solving a discretized Laplace equation by a Red-Black Gauss-Seidel iteration. Electrode contacts were modeled as arrays of linked point current sources and the boundaries of the model were grounded. The transmembrane potentials generated in individual nerve fibers within the fascicle were determined using a McNeal-type model of a myelinated nerve fiber [5].

A monofascicular nerve with a diameter of about $0.7 \mathrm{~mm}$, surrounded by a $50 \mu \mathrm{m}$ perineurium and $50 \mu \mathrm{m}$ layer of epineurium, was modeled (Fig. 1). This is comparable to the largest of the several fascicles typically found in the rabbit peroneal nerve. A total nerve length of $23 \mathrm{~mm}$ was modeled. The electrode consisted of a cylindrical insulating cuff $(1.25 \mathrm{~mm}$ i.d., $0.25 \mathrm{~mm}$ thick, $11 \mathrm{~mm}$ long) with three $1.0 \mathrm{~mm}$ wide circular contacts spaced $3.0 \mathrm{~mm}$ apart. The nerve and cuff were surrounded by a layer of saline, and the outermost layer of the model was a lower conductivity boundary layer to simulate a distant ground. The central contact of the electrode served as a cathode, and the outer two contacts as anodes. The current through each anode was half of the current through the cathode, so that the sum of the anodal and cathodal currents was zero. $500 \mu \mathrm{s}$ stimulus pulses were used.

\section{RESULTS}

During tripolar stimulation, a nerve fiber is typically excited to produce an action potential (AP) in the region under the cathode. The AP then propagates away from the cathode in both directions. Propagation of the AP under
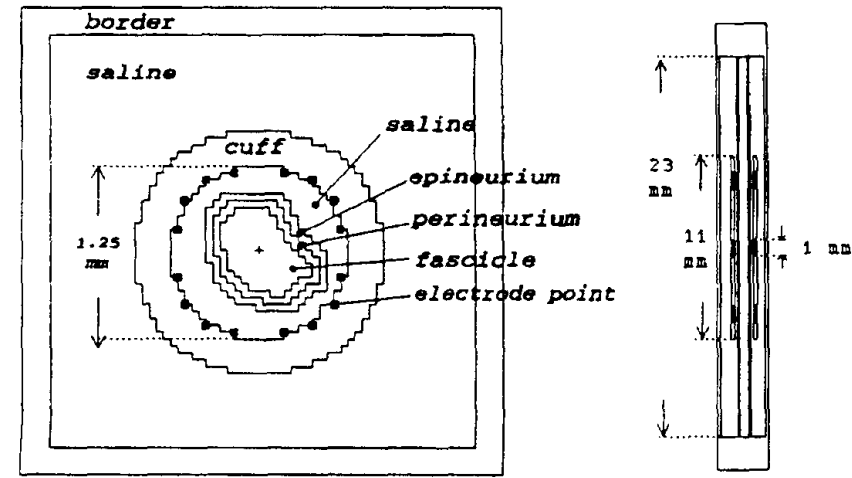

Figure 1: Transverse and longitudinal sections of model. + indicates location of nerve fiber.

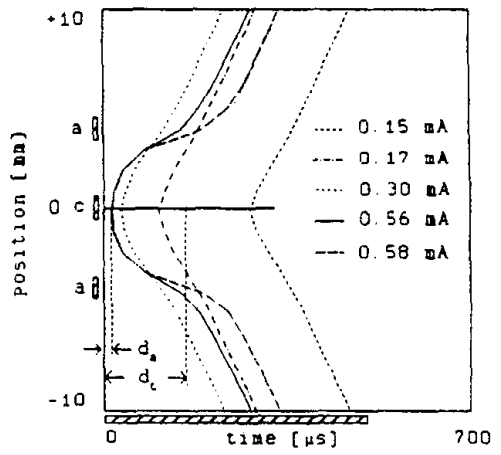

Figure 2: Action potential location vs. time. Hatched bar shows stimulus pulse duration; $a$ and $c$ indicate anode and cathode positions. 
Figure 3

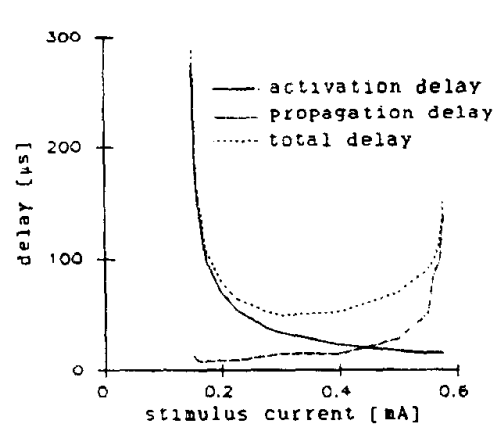

Figure 4

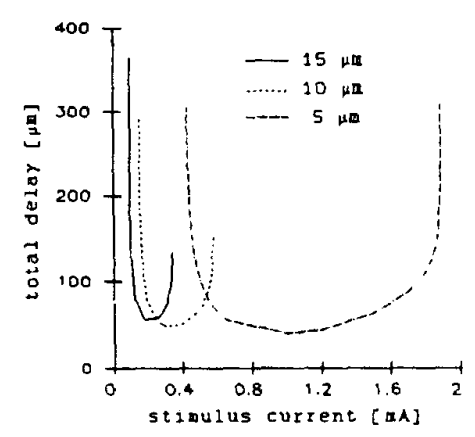

Figure 3: Activation delay (d), total delay (d) and propagation delay $\left(\mathbf{d}_{1}-\mathbf{d}_{2}\right)$ vs. stimulus current, for $10 \mathrm{~mm}$ fiber.

Figure 4: Total delay plotted as fuction of stimulus current for 5 $\mu \mathrm{m}, 10 \mu \mathrm{m}$ and $15 \mu \mathrm{m}$ fibers. the anodes may be slowed or blocked entirely depending on the amplitude of the stimulus pulse. Once the AP has travelled beyond the anode, it will conduct along the nerve fiber at a constant velocity. We plotted the position of the AP relative to the cathode as a function of time. The position of the AP was defined as the point where the rising phase of the potential was $50 \mathrm{mV}$.

In Fig. 2, the positions of APs in a $10 \mu \mathrm{m}$ fiber are plotted for several stimulus amplitudes. Near the excitation threshold $(0.15 \mathrm{~mA})$, the AP travels away from the cathode at roughly a constant conduction velocity. There is an activation delay of $325 \mu \mathrm{s}$ before the AP is produced under the cathode. Near the blocking threshold $(0.58 \mathrm{~mA})$, the AP appears much earlier and travels quickly near the cathode because multiple nodes are depolarized by the cathodal current. However, propagation is delayed under the anodes. These effects result in the non-linearity of the position-time plot. The arrival time of the AP at a distant point on the nerve will equal the distance travelled divided by the normal conduction velocity, plus a total delay term which includes the activation delay, anodal delay and cathodal acceleration effect. The activation delay $\left(d_{1}\right)$ and total delay $\left(\mathrm{d}_{1}\right)$ for the $0.58 \mathrm{~mA}$ stimulus pulse are indicated in Fig. 2.

Fig. 3 shows $d_{a}, d_{1}$, and the propagation delay $\left(d_{1}-d_{2}\right)$ for a $10 \mu \mathrm{m}$ fiber plotted versus stimulus amplitude. At the excitation and blocking thresholds, $\mathrm{d}$ increases rapidly. I $\mathrm{n}$ Fig. 4 , the $d$ is plotted vs. stimulus current for 5,10 and $15 \mu \mathrm{m}$ fibers to illustrate the effect of fiber diameter.

\section{Discussion}

Our results indicate that small fibers can be excited while larger fibers are blocked, in agreement with previous simulation and experimental results, and furthermore that activation and propagation delay is dependent on fiber diameter and stimulus amplitude. With the contact spacing we used, increasing the pulse duration beyond $500 \mu \mathrm{s}$ would not have a significant effect because by the end of the pulse the action potentials of all the fibers have travelled beyond the area effected by the anodal current before the end of the pulse. If a shorter pulse was used, anodal effects would presumably be reduced. In this study we have considered only the effect of fiber diameter, but excitation and block- ing thresholds will be lower for fibers at the edge of the fascicle than at the center, so the influence of fiber position must also be investigated before the compound response of the fiber population in a fascicle to tripolar stimulation can be fully understood.

\section{CONCLUSIONS}

The results of our simulations predict that tripolar stimulation is capable of producing selective activation of fibers of different sizes, but with distinct temporal effects. A clear relationship exists between stimulus amplitude, fiber diameter, and activation and propagation delay. We anticipate that this information will aid in the interpretation of tripolar neural stimulation effects. By expressing the retationship in mathematical terms, and also taking into account the influence of fiber position, the pattern of neural activity produced by stimulation with a tripolar cuff electrode could be predicted. The relationship could also be incorporated into algorithms for compound action potential decomposition.

\section{REFERENCES}

[1] C. van den Honert and J.T. Mortimer, "A Technique for Collision Block of Peripheral Nerve: Single Stimulus Analysis," IEEE Trans. Biomed. Engr., vol. BME28, pp. 373-378, 1981.

[2] Z.P. Fang and J.T. Mortimer, "Selective Activation of Small Motor Axons by Quasitrapezoidal Current Pulses," IEEE Trans. Biomedical Eng., vol. 38, pp.168-174, 1991.

[3] J. Holsheimer, J.J. Struijk and G. G. van der Heide, "Electrical Stimulation of Myelinated Nerve Fibers: a Modelling Study," Proc. 3rd Vienna Workshop on Functional Electrical Stimulation, pp.151-154, 1989.

[4] J.J. Struijk, J. Holsheimer, G.G. van der Heide and H.B.K. Boom, "Recruitment of Dorsal Column Fibers in Spinal Cord Stimulation, "IEEE Trans. Biomed. Engr., vol. BME-39, pp. 903-912, 1992.

[5] D.R. McNeal, "Analysis of a model for excitation of myelinated nerve," IEEE Trans. Biomed. Engr., vol. BME-23, pp. 329-337, 1976. 